\title{
Perpetración y victimización de la violencia en relaciones de parejas en jóvenes que cursan educación superior en la ciudad de Osorno, Chile
}

\author{
Ramón Vivanco Muñoz
}

Universidad de Los Lagos, Osorno, Chile.

Email: rvivanco@ulagos.cl

\section{Soraya Espinoza Moraga \\ Universidad de Los Lagos, Osorno, Chile. \\ Email: sespinoza@ulagos.cl}

\section{Cristian Romo Tregear}

Universidad de Los Lagos, Osorno, Chile. Email: cromo@ulagos.cl

\section{Alex Véliz Burgos}

Universidad de Los Lagos, Osorno, Chile.

Email: alex.veliz@ulagos.cl

\section{Antonio Vargas Peña}

Universidad de Los Lagos, Osorno, Chile.

Email: antoniovargaspena@gmail.com

Resumen: La presente investigación tuvo por objetivo conocer la existencia de manifestaciones de violencia en relaciones de pareja en jóvenes que cursan educación superior en la ciudad de Osorno, Chile. Los participantes fueron 360 estudiantes ( $M=23,13$ años y $\mathrm{DT}=4,4$ años) a quienes se aplicó la Escala de Maltrato en la Pareja Forma A (Rey, 2009), cuyo requisito fue estar o haber estado en una relación de pareja en los últimos doce meses. Los resultados obtenidos muestran que un $85 \%$ de los participantes declaró haber recibido algún tipo de violencia, sin diferencia significativa entre sexos. Sin embargo, en violencia ejercida existe diferencia estadísticamente significativa (mujeres 88,4\% y hombres 80,9\%). Los datos muestran la gravedad del fenómeno sin diferencia de género, lo cual se transforma en un desafío para las políticas públicas y para los programas de intervención.

Palabras clave: Violencia de pareja, victimización, perpetración, estudiantes universitarios. 


\title{
Perpetration and victimization of violence in young couples studying higher education in the city of Osorno, Chile
}

\begin{abstract}
The present investigation aimed to reveal the existence of manifestations of violence in couple relationships in young people pursuing higher education in the city of Osorno, Chile. Participants were 360 students ( $M=23,13$ years and SD $=4,4$ years) on whom an Abuse on Couple Scale in Form A (Rey, 2009), was applied, where the requirement was to be or had been in a partner relationship in the last twelve months. The results show that $85 \%$ of the participants reported receiving some form of violence, with no significant difference between sexes. However, in exercised violence there existed a statistically significant difference (88.4\% women and $80.9 \%$ men). The data shows the gravity of the phenomenon without gender difference, which becomes a challenge for public policies and intervention programs.
\end{abstract}

Keywords: Dating violence, victimization, perpetration, college students.

\section{Perpetração e vitimização da violência nas relações de casais jovens que cursam o ensino superior na cidade de Osorno, Chile}

Resumo: Este trabalho teve como objetivo revelar a existência de manifestações de violência nas relações de jovens que cursam o ensino superior na cidade de Osorno, Chile. Os participantes foram 360 estudantes ( $M=23,13$ anos e DP $=4,4$ anos), aos quais foi aplicada a Escala de Maus-tratos no casal Forma A (Rey, 2009), cujo requisito foi estar e ter mantido um relacionamento nos últimos doze meses. Os resultados mostram que $85 \%$ dos participantes relataram ter recebido algum tipo de violência, não havendo diferença significativa entre os sexos. No entanto, na violência exercida existe uma diferença estatisticamente significativa (88,4\% mulheres e $80,9 \%$ homens). Os dados mostram a gravidade do fenómeno sem diferença de gênero, o qual se torna um desafio para as políticas públicas e os programas de intervenção.

Palavras-chave: violência entre casais, vitimização, perpetração, estudantes universitários.

\section{Introducción $^{1}$}

La violencia en las relaciones de pareja es uno de los problemas sociales más serios con los que se enfrenta la sociedad. Las investigaciones realizadas en la problemática se han concentrado principalmente en las relaciones entre parejas adultas, siendo escasos los antecedentes sobre la violencia en las parejas jóvenes, independiente de la estabilidad y duración del vínculo.

Puget y Berenstein (1998) definen la relación de pareja como una conexión o correspondencia en una estructura vincular de dos personas de distinto sexo cuyos parámetros definitorios son: una cotidianidad que se designa como la estabilidad basada en una unidad temporal y espacial ca- 
racterizada por los intercambios diarios, un proyecto vital compartido, el cual es la realización o logros ubicados en la dimensión de tiempo futuro y la concreción de relaciones sexuales, la cual corresponde a la necesidad de un otro que está ligada a la aceptación de incompletud o tendencia monogámica.

Es en las primeras relaciones amorosas donde jóvenes construyen sus ideas iníciales sobre qué esperan de una relación de pareja y cómo se debe comportar en la intimidad, algo que a futuro va a repercutir en su vida adulta (Dion y Dion 1993; Furman y Flanagan, 1997).

Los resultados investigativos indican que es fundamental que estas primeras experiencias sean positivas, lo cual no siempre sucede como se espera. El "Estudio de violencia en las relaciones de pareja entre jóvenes” (SERNAM, 2009) menciona que en el pololeo, los niveles de violencia son semejantes entre ambos sexos; sin embargo estas características cambian en la relación de convivencia o matrimonio, pasando a ser la mujer la que es mayoritariamente objeto de violencia por parte de su pareja. Una de las hipótesis planteadas señalaba que en el pololeo la mujer establecía relaciones de mayor igualdad y grados de libertad que ya en una etapa de adultez o mayor formalidad de la relación se perdían o anulaban.

En el marco del Seminario “Amor sin violencia. Prevención en parejas jóvenes”, SERNAM (2012) se entregaron datos cualitativos sobre el fenómeno, entre estos destacan que un alto porcentaje de jóvenes ha experimentado violencia en sus relaciones de pareja. En relación a cómo ven este problema, se señalo que existe una diferencia por nivel socio-económico, siendo los jóvenes de sectores más bajos quienes ven la violencia como más real, considerándola como habitual, en cambio los jóvenes de los sectores socio-económicos más altos presentan un discurso más conceptual con una menor visibilización de su presencia en su ambiente más íntimo. En relación al reconocimiento de la violencia, esta es más compleja para el hombre. Sobre la violencia cruzada se indicó que es transversal a los diferentes niveles socio-económicos, “...los dos pegan igual. Se reconocería que la mujer, ya no se deja agredir fácilmente, tendería a defenderse” (Ibid:17). Sobre las razones que llevan a los jóvenes a presentar conductas violentas se tendrían las alteraciones de tipo psicológica y por un aprendizaje de conductas de los propios padres.

El estudio de Vizcarra y Póo (2011) sobre violencia de parejas en estudiantes universitarios del sur de Chile, concluyo que un 57\% de los entrevistados reportó haber vivido violencia psicológica alguna vez en su vida y un 26\% violencia física. El análisis permitió determinar factores asociados a la presencia de violencia física y psicológica. Los factores asociados a violencia psicológica fueron: sexo, violencia física recibida, actitudes favorables hacía la violencia y mayor tiempo de relación de pareja. La violencia física se asoció a sexo, violencia psicológica recibida, actitudes favorables hacia la violencia y baja participación religiosa. 
Kanin (1957) alertó por primera vez sobre la existencia de conductas violentas en las relaciones de parejas jóvenes. En su estudio retrospectivo encontró que un 30\% de las mujeres había sufrido agresiones sexuales a manos de sus parejas. El estudio desarrollado en España por González y Santana (2001) estableció que un 7,5\% de hombres y un 7,1\% de mujeres jóvenes reconocían haber empujado o pegado a sus parejas en una o más ocasiones. Serran y Firestone (2004) señalan que el uso de la violencia no suele surgir de forma espontánea durante la vida de pareja o el matrimonio; con frecuencia se inicia durante el noviazgo en jóvenes y adolescentes.

\section{Tipos de violencia}

Las formas de violencia en pareja están dadas preferentemente por la violencia física, la cual corresponde al abuso corporal. Como señalan Alberdi y Matas (2002) corresponde a una escalada que es progresiva y que puede comenzar con un empujón o pellizco y llegar a lesiones graves y en casos extremos al homicidio, la violencia psíquica o psicológica, que se asocia a la desvalorización de la otra persona (Ibid: 2002). Algunos de los comportamientos a través de la cual se manifiesta son la ridiculización, amenazas verbales e insultos, humillación; acciones que afectan la autoestima y el bienestar psicológico de la persona (Follingstad, Rutledge, Berg, Hause, y Polek, 1990). La violencia sexual, se da "mediante presiones físicas o psíquicas que imponen relaciones sexuales no deseadas mediante coacción, intimidación o indefensión” (Alberdi y Matas, 2002: 94). La violencia emocional corresponde a "cualquier acto de naturaleza verbal o no verbal que provoca intencionalmente en la victima una reacción de ansiedad, temor o miedo, como las intimidaciones y las amenazas; incluye los actos de violencia dirigidos a un familiar o a un conocido de la víctima, a sus bienes o hacia el agresor mismo, realizados con el mismo fin” (Rey-Anacona, 2013:146). La violencia económica se asocia a la intención de "forzar a la otra persona a depender económicamente del agresor, no dejándola trabajar o por otros medios; ejercer control sobre los recursos financieros de la víctima o explotarla sexualmente” (Ibid:146).

Otras líneas investigativas han complementado esta tipología de violencia. El trabajo de Johnson (2011) distingue cuatro tipos de violencia en la pareja: El primero es el terrorismo íntimo, el cual corresponde a un patrón de violencia coercitiva con el que la mayoría de la gente suele asociar a la violencia doméstica. En palabras de Johnson "involucra la combinación de violencia física y/o sexual con una variedad de tácticas de control no violentas, cómo el abuso económico, el uso de los hijos, amenazas e intimidación, invocación del privilegio masculino, vigilancia constante, culpabilización de la víctima, amenazas de acusación a las autoridades de inmigración, o amenazas de echar a una persona al trabajo o de la familia” (Johnson, 2011:290). El segundo corresponde a la resistencia violenta: es la reacción al terrorismo íntimo. Para algunos, es una respuesta instintiva a la violencia sistemática por parte de la pareja. Para otros, no ocurre sino luego 
de que la víctima se da cuenta de que la violencia seguirá ocurriendo para siempre si algo no la detiene. El tercero es la violencia situacional de pareja: Este tipo de violencia no se considera dentro de un patrón de control coercitivo, sino que más bien se produce cuando conflictos en la pareja derivan en una discusión que llega a agresiones que se vuelven violentas. Es ocasional y el más común de los tipos de violencia de pareja. A diferencia del terrorismo íntimo no está relacionado con un intento de control coercitivo de la pareja. Es más o menos simétrico en términos de perpetración por género. El cuarto tipo es el control violento mutuo, el cual consistiría en un patrón de abuso en que ambos miembros de la pareja son controladores y violentos, es decir, donde hay dos terrorismos íntimos puestos en combate por hacerse del control de la pareja. Es el menos frecuente de los tipos de violencia, apareciendo en un número muy reducido de casos.

Desde mediados de los años 70, las investigaciones sobre violencia en relaciones de pareja han señalado que la perpetración de conductas violentas por parte de las mujeres hacia los hombres es igual e incluso mayor a las reportadas por hombres hacia las mujeres (Archer, 2000). Estos hallazgos han sido permanente fuente de polémicas al contradecir, al menos en apariencia, la perspectiva de género, donde es principalmente el hombre quien ejerce una violencia sistemática contra la mujer en defensa de una estructura familiar patriarcal. Además de la conformación de las muestras, los instrumentos de recolección de información utilizados en estos estudios han sido parte central del debate respecto a la investigación en violencia de pareja. La violencia contra la mujer está estrechamente relacionada con la desigualdad de género. La violencia contra las mujeres tiene su origen en el patriarcado, que es una forma de dominio y organización social compartida por casi todas las sociedades conocidas históricamente y que solo recientemente empieza a cuestionarse. Maturana (1997) dice que la agresión y la violencia no son aspectos biológicos del ser humano. La agresión y la violencia surgen como modos culturales de vivir en un contexto de patriarcado, por lo que son experimentados socialmente por los individuos.

Así la presencia de violencia en las relaciones de parejas en jóvenes pasa a ser un problema de índole social el cual amerita ser conocido más integralmente y más globalmente, lo cual llevo al equipo investigador a plantearse el objetivo de "Conocer la existencia de manifestaciones de violencia en relaciones de pareja en jóvenes que cursan educación superior en la ciudad de Osorno, Chile”.

\section{Método}

\section{Participantes}

El universo estuvo constituido por 6256 estudiantes de cuatro centros de educación superior de la ciudad de Osorno; la Universidad de Los 
Lagos, la Universidad Tecnológica de Chile, el Instituto Profesional La Araucana y el Instituto Tecnológico Regional de la Universidad de Los Lagos, de los cuales se seleccionaron los sujetos a través de un muestreo incidental con 360 casos en dos etapas. En la primera se estratifico el número de sujetos de estudio según el número de matrícula en los establecimientos de educación superior. En una segunda etapa se seleccionaron los sujetos. Los estudiantes presentaron una media de 23,13 años y una desviación típica de 4,4 años.

Tabla 1. Distribución de estudiantes por género e institución

\begin{tabular}{|l|c|c|c|}
\hline Institución & Hombres & Mujeres & Total \\
\hline Universidad de Los Lagos & 62 & 132 & 194 \\
\hline Universidad Tecnológica de Chile & 17 & 11 & 28 \\
\hline Instituto Profesional La Araucana & 22 & 33 & 55 \\
\hline Instituto Tecnológico Regional de la Universidad de Los Lagos & 25 & 48 & 83 \\
\hline Total & 136 & 224 & 360 \\
\hline
\end{tabular}

Fuente: Elaboración Propia.

\section{Instrumento}

Para medir específicamente la prevalencia de situaciones de violencia en las relaciones de pareja, se utilizó una versión propia de la Lista de chequeo de experiencias de maltrato en la pareja -Forma A (Rey, 2009). La versión utilizada en esta investigación fue una escala de autoreporte, compuesta por una lista de 68 ítems referidos a cinco tipos de violencia en relaciones de pareja: psicológica, emocional, física, económica y sexual. Las respuestas fueron presentadas como escala tipo Likert con las opciones "Nunca”, "Una vez", “Algunas veces” y "Muchas veces”. Se agregó el ítem "Registró tu (registraste su) celular sin tu consentimiento" como forma de maltrato psicológico. Para medir tanto perpetración como victimización, cada ítem tuvo una doble entrada referida a la percepción de maltrato tanto ejercido como recibido respecto a la pareja.

A continuación se presentan los valores alfa de Cronbach para cada una de las dimensiones de violencia analizada. 
Tabla 2. Análisis de confiabilidad de Subescalas.

\begin{tabular}{|l|c|}
\hline \multicolumn{1}{|c|}{ Subescalas } & $\begin{array}{c}\text { alfa de } \\
\text { Cronbach }\end{array}$ \\
\hline Violencia recibida (escala total) & 0,95 \\
\hline Violencia psicológica recibida & 0,933 \\
\hline Violencia Emocional recibida & 0,772 \\
\hline Violencia Física recibida & 0,719 \\
\hline Violencia Económica recibida & 0,523 \\
\hline Violencia Sexual recibida & 0,513 \\
\hline Violencia ejercida (escala total) & 0,937 \\
\hline Violencia psicológica ejercida & 0,918 \\
\hline Violencia Emocional ejercida & 0,72 \\
\hline Violencia Física ejercida & 0,716 \\
\hline Violencia Económica ejercida & 0,575 \\
\hline Violencia Sexual ejercida & 0,649 \\
\hline
\end{tabular}

Fuente: Elaboración propia.

Se observa en la tabla anterior que la confiabilidad de las subescalas va de ,513 a ,937. Lo que según Nunnally (1987) es adecuado, pues para efectos de investigación pueden utilizarse escalas con valores mínimos de fiabilidad de ,50 en adelante.

\section{Procedimiento}

Las etapas de implementación de la investigación estuvieron dadas por una primera entrevista con los Directores/Rectores y/o encargados de Asuntos Estudiantiles de los Centros de Educación Superior a las cuales se les solicito el acceso a las unidades académicas. Posteriormente se tomó contacto con los Jefes/Coordinadores de Carreras y luego con los estudiantes. Se aplicó un filtro en la selección de los estudiantes dado por la mantención de algún tipo de relación de pareja en los últimos 12 meses. Los participantes encuestados fueron contactados en las salas de clases por mediación de profesores a los que previamente se había planteado el tema de estudio o bien dentro de espacios físicos (bibliotecas, salas de estudio, patios internos, etc.) de las instituciones en las que se realizó la investigación. Los encargados de entregar los cuestionarios y supervisar su aplicación fueron estudiantes de Trabajo Social de la Universidad de Los Lagos contactados y capacitados por los investigadores a cargo del estudio. Al momento de entregar los cuestionarios se aseguró el carácter voluntario y anónimo del estudio. Cada estudiante firmó una carta de consentimiento informado. El tiempo de respuesta promedio de los cuestionarios fue de 30 minutos. 


\section{Análisis Estadísticos}

Los análisis de los datos se realizaron en dos momentos: el primero buscó verificar la confiabilidad del instrumento en la muestra de estudiantes a través de la prueba de consistencia interna alfa de Cronbach. Un segundo momento se relacionó con análisis de frecuencias de las respuestas de los participantes, la prueba X2 para verificar relaciones estadísticas entre las respuestas de los participantes de acuerdo al género. Se incorporó además la prueba t de Student para verificar diferencias en los promedios de respuestas de los participantes según género en las diferentes dimensiones. Finalmente se utiliza la prueba r de Pearson para establecer la existencia de relaciones entre las dimensiones de la violencia evaluadas.

\section{Resultados}

Los resultados nos muestran que la prevalencia general de haber recibido algún tipo de violencia es de $85.3 \%$ de los participantes $(n=307)$, los cuales reportaron recibir al menos en una ocasión algún tipo de violencia en su relación de pareja. Entre los varones, un 83,1\% (n=113) reportó esta situación, mientras que en las mujeres, ese porcentaje fue de $86,6 \%(n=194)$. No existe diferencia estadística entre hombres y mujeres.

Los resultados en cuanto a violencia ejercida nos indican que un 85,6\% ( $n=308)$ de los participantes señaló ejercer al menos algún tipo de violencia. El porcentaje de mujeres en este caso alcanzó un 88,4\% ( $\mathrm{n}=198)$ frente a un $80,9 \%$ de los hombres $(n=110)$, siendo esta diferencia significativa $(\mathrm{X} 2=3,863 ; \mathrm{p}=0,049)$. 
Tabla 3. Porcentaje de participantes que fueron objeto de violencia por lo menos una vez

\begin{tabular}{|c|c|c|c|c|c|}
\hline $\begin{array}{l}\text { Tipo de } \\
\text { Violencia } \\
\text { Recibida }\end{array}$ & Sexo & $\mathrm{N}$ & $\%$ & $X^{2}$ & $\mathrm{P}$ \\
\hline \multirow{3}{*}{ Psicológico } & Hombres & 112 & $82,4 \%$ & \multirow{3}{*}{1,201} & \multirow{3}{*}{, 273} \\
\hline & Mujeres & 194 & $86,6 \%$ & & \\
\hline & Total & 306 & $85,0 \%$ & & \\
\hline \multirow{3}{*}{ Emocional } & Hombres & 34 & $25,0 \%$ & \multirow{3}{*}{4,489} & \multirow{3}{*}{, 034} \\
\hline & Mujeres & 80 & $35,7 \%$ & & \\
\hline & Total & 114 & $31,7 \%$ & & \\
\hline \multirow{3}{*}{ Fisico } & Hombres & 22 & $16,2 \%$ & \multirow{3}{*}{5,830} & \multirow{3}{*}{, 016} \\
\hline & Mujeres & 61 & $27,2 \%$ & & \\
\hline & Total & 83 & $23,1 \%$ & & \\
\hline \multirow{3}{*}{ Económico } & Hombres & 15 & $11,0 \%$ & \multirow{3}{*}{, 052} & \multirow{3}{*}{, 820} \\
\hline & Mujeres & 23 & $10,3 \%$ & & \\
\hline & Total & 38 & $10,6 \%$ & & \\
\hline \multirow{3}{*}{ Sexual } & Hombres & 12 & $8,8 \%$ & \multirow{3}{*}{3,847} & \multirow{3}{*}{, $050^{\circ}$} \\
\hline & Mujeres & 36 & $16,1 \%$ & & \\
\hline & Total & 48 & $13,3 \%$ & & \\
\hline
\end{tabular}

Fuente: Elaboración propia.

Como se observa en la tabla 3 se destaca una alta prevalencia del fenómeno, el reporte fue mayor en las mujeres en todos los tipos de violencia, salvo la violencia económica que los hombres reportaron una mayor prevalencia que las mujeres de recibir este tipo de violencia por parte de sus parejas.

La comparación realizada entre los porcentajes entre mujeres y hombres por medio de la prueba estadística X2 de Pearson, (Tabla 3) reportó diferencias estadísticamente significativas solo para la violencia emocional donde las mujeres reportan su presencia en un 35,7\% y los hombres en un $25 \%$ (X2=4,489, $\mathrm{p}=, 034)$, física $27,2 \%$ de mujeres y 16,2\% de hombres lo 
reportan $(\mathrm{X} 2=5,830, \mathrm{p}=, 016)$ y sexual, $16,1 \%$ de mujeres y $8,8 \%$ de hombres describen su ocurrencia $(\mathrm{X} 2=3,847, \mathrm{p}=, 050)$. En la violencia económica y la psicológica si bien ambos géneros reportan haber sido objeto de ella, no se encontraron diferencias estadísticamente significativas entre estudiantes mujeres y hombres.

Tabla 4 Porcentaje de participantes que ejercieron, a lo menos una vez algún tipo de violencia y su diferencia por género.

\begin{tabular}{|c|c|c|c|c|c|}
\hline Tipo de violencia ejercida & Sexo & $\mathrm{N}$ & $\%$ & $X^{2}$ & $P$ \\
\hline \multirow{3}{*}{ Psicológico } & Hombres & 107 & $78,7 \%$ & \multirow{3}{*}{4,944} & \multirow{3}{*}{, $026^{\circ}$} \\
\hline & Mujeres & 196 & $87,5 \%$ & & \\
\hline & Total & 303 & $84,2 \%$ & & \\
\hline \multirow{3}{*}{ Emocional } & Hombres & 21 & $15,4 \%$ & \multirow{3}{*}{4,219} & \multirow{3}{*}{, $040^{*}$} \\
\hline & Mujeres & 55 & $24,6 \%$ & & \\
\hline & Total & 76 & $21,1 \%$ & & \\
\hline \multirow{3}{*}{ Físico } & Hombres & 17 & $12,5 \%$ & \multirow{3}{*}{14,961} & \multirow{3}{*}{, $000^{\circ}$} \\
\hline & Mujeres & 68 & $30,4 \%$ & & \\
\hline & Total & 85 & $23,6 \%$ & & \\
\hline \multirow{3}{*}{ Económico } & Hombres & 4 & $2,9 \%$ & \multirow{3}{*}{2,847} & \multirow{3}{*}{, 092} \\
\hline & Mujeres & 16 & $7,1 \%$ & & \\
\hline & Total & 20 & $5,6 \%$ & & \\
\hline \multirow{3}{*}{ Sexual } & Hombres & 9 & $6,6 \%$ & \multirow{3}{*}{, 019} & \multirow{3}{*}{, 890} \\
\hline & Mujeres & 14 & $6,3 \%$ & & \\
\hline & Total & 23 & $6,4 \%$ & & \\
\hline
\end{tabular}

$\mathrm{P}<0,05$

Fuente: Elaboración propia.

En cuanto a la comparación realizada por género en cada tipo de violencia, las mujeres reconocen mayor perpetración o ejercicio de la violencia que los hombres en violencia psicológica, emocional, física y económica. En la violencia sexual en cambio, los hombres reconocen una mayor prevalencia. No obstante, por medio de la prueba estadística X2 de Pearson, (Tabla 4) se reportaron diferencias estadísticamente significativas solo para 
la violencia psicológica, donde las mujeres la señalan en un $87,5 \%$ y los hombres en un 78,7\% (X2=4,944 p=,026), violencia emocional, un $24,6 \%$ de las mujeres la reportan y $15,4 \%$ de los hombres $(X 2=4,219, \mathrm{P}=, 040)$ y física, un $30,4 \%$ de las mujeres señalaron ejercerla y $12,5 \%$ de los hombres $(\mathrm{X} 2=14,961, \mathrm{p}=, 000)$. No se encontró diferencias estadísticamente significativas la para violencia sexual y económica entre hombres y mujeres.

Tabla 5 Frecuencia de cada tipo de violencia y su diferencia por género.

\begin{tabular}{|c|c|c|c|c|}
\hline Violencia & Sexo & Media & $\begin{array}{c}\text { Desviación } \\
\text { típ. }\end{array}$ & $\mathrm{p}$ \\
\hline \multirow[t]{2}{*}{ Psicológica Recibida } & Mujer & 15,15 & 17,35 & \\
\hline & Hombre & 12,09 & 12,76 & \\
\hline \multirow[t]{2}{*}{ Psicológica Ejercida } & Mujer & 12,79 & 13,35 & \multirow{2}{*}{0,01} \\
\hline & Hombre & 8,47 & 8,47 & \\
\hline \multirow[t]{2}{*}{ Emocional Recibida } & Mujer & 1,60 & 3,83 & \multirow{2}{*}{0,01} \\
\hline & Hombre & 0,71 & 1,61 & \\
\hline \multirow[t]{2}{*}{ Emocional Ejercida } & Mujer & 0,86 & 2,71 & \\
\hline & Hombre & 0,45 & 1,21 & \\
\hline \multirow[t]{2}{*}{ Física Recibida } & Mujer & 0,73 & 1,62 & \multirow{2}{*}{0,001} \\
\hline & Hombre & 0,34 & 0,98 & \\
\hline \multirow[t]{2}{*}{ Física Ejercida } & Mujer & 0,77 & 1,61 & \multirow{2}{*}{0,01} \\
\hline & Hombre & 0,26 & 0,78 & \\
\hline \multirow[t]{2}{*}{ Económica Recibida } & Mujer & 0,24 & 0,88 & \\
\hline & Hombre & 0,16 & 0,49 & \\
\hline \multirow[t]{2}{*}{ Económica Ejercida } & Mujer & 0,15 & 0,65 & \\
\hline & Hombre & 0,05 & 0,30 & \\
\hline \multirow[t]{2}{*}{ Sexual Recibida } & Mujer & 0,36 & 0,99 & \multirow{2}{*}{0,02} \\
\hline & Hombre & 0,15 & 0,52 & \\
\hline \multirow[t]{2}{*}{ Sexual Ejercida } & Mujer & 0,15 & 0,76 & \\
\hline & Hombre & 0,14 & 0,53 & \\
\hline
\end{tabular}

$\mathrm{P}<0,05$

Fuente: Elaboración propia.

Al hacer un análisis general de la frecuencia reportada por tipo de violencia, se encuentra que, en todas las dimensiones observadas, las mujeres obtienen un mayor promedio que los hombres. Es decir, las mujeres del estudio reportan tanto recibir como ejercer una mayor frecuencia de conductas violentas en sus relaciones de pareja que los hombres.

Al compararse los promedios de respuesta de los estudiantes universitarios por tipo de violencia y género mediante la prueba t de Student, se encuentran diferencias estadísticamente significativas en las dimensiones de violencia psicológica ejercida, emocional recibida, física recibida, física ejercida y sexual recibida, en todas la cuales las mujeres obtuvieron un promedio de reporte mayor que los hombres. 
Observando los tipos de violencia que muestran diferencias estadísticamente significativas, las mujeres tienden a reportar el recibir más violencia por parte de sus parejas, como también a reconocer más que los hombres conductas de ejercidas por ellas en sus relaciones. En el caso de la violencia física, esta diferencia es significativa en ambas direcciones, donde las mujeres reconocen tanto recibir ( $\mathrm{M}=, 73$ las mujeres y ,34 los hombres, $\mathrm{p}<0,001$ ) como ejercer ( $\mathrm{M}=, 77$ mujeres $\mathrm{y}, 26$, los hombres, $\mathrm{p}<0,01$ ) más agresiones físicas de parte de o hacia sus parejas que los hombres.

Como se observa en la tabla anterior, existen diferencias estadísticamente significativas en las dimensiones violencia psicológica ejercida donde el puntaje promedio de las mujeres es más alto que el de los hombres ( $M=12,78$ y 8,47, respectivamente, $p<0,001$ )

En el caso de la violencia emocional, las mujeres reportan recibir más este tipo de violencia que los hombres ( $M=1,60$ para las mujeres, $M=, 71$ para los hombres; $\mathrm{p}<0,01$ ), no encontrándose una diferencia significativa respecto a la violencia ejercida.

La dimensión de violencia económica es la única en que no se encuentran diferencias significativas entre géneros tanto en forma recibida como ejercida.

En el caso de la violencia sexual recibida, las mujeres obtienen una media significativamente mayor que los hombres $(\mathrm{M}=, 36 \mathrm{y}, 15$ de los hombres, $\mathrm{p}<0,2)$. Por contraste, no se obtuvo diferencias estadísticamente significativas entre géneros respecto a la violencia sexual ejercida.

Tabla 6. Asociaciones entre distintos tipos de violencia tanto recibida como ejercida

\begin{tabular}{|c|c|c|c|c|c|c|c|c|c|}
\hline Violencia & $\begin{array}{c}\text { Psicológica } \\
\text { E }\end{array}$ & $\begin{array}{c}\text { Emocional } \\
\mathrm{R}\end{array}$ & $\begin{array}{c}\text { Emocional } \\
\mathrm{E}\end{array}$ & $\begin{array}{c}\text { Física } \\
\mathrm{R}\end{array}$ & $\begin{array}{c}\text { Física } \\
\mathrm{E}\end{array}$ & \begin{tabular}{|c} 
Económica \\
$\mathrm{R}$
\end{tabular} & $\begin{array}{c}\text { Económica } \\
\text { E }\end{array}$ & $\begin{array}{c}\text { Sexual } \\
\mathrm{R}\end{array}$ & $\begin{array}{c}\text { Sexual } \\
\mathrm{E}\end{array}$ \\
\hline $\begin{array}{c}\text { Psicológica } \\
\text { R }\end{array}$ &, $753^{* *}$ & ,691" & ,503"* &, $729^{* *}$ &, $509^{*}$ & ,548* &, $277^{* *}$ &, $525^{* *}$ &, $295^{* *}$ \\
\hline $\begin{array}{c}\text { Psicológica } \\
\text { E }\end{array}$ & 1 & ,613" & ,632* & ,609* &, $640^{* *}$ &, $364^{* *}$ &, $386^{* *}$ &, $437^{* *}$ &, $340^{* *}$ \\
\hline $\begin{array}{c}\text { Emocional } \\
\mathrm{R}\end{array}$ & & 1 &, $740^{* *}$ &, $763^{* *}$ &, $579^{* *}$ &, $548^{* *}$ &, $581^{* *}$ &, $576^{* *}$ & ,499** \\
\hline $\begin{array}{c}\text { Emocional } \\
\text { E }\end{array}$ & & & 1 & ,610" &, $633^{* *}$ & , 481"* &, $736^{* *}$ &, $421^{* *}$ &, $645^{* *}$ \\
\hline $\begin{array}{c}\text { Física } \\
\mathrm{R}\end{array}$ & & & & 1 &, $653^{* *}$ &, $492^{* *}$ &, $411^{* *}$ &, $440^{* *}$ &, $292^{* *}$ \\
\hline $\begin{array}{c}\text { Física } \\
\mathrm{E}\end{array}$ & & & & & 1 & ,290* &, $436^{* *}$ &, $332^{* *}$ &, $323^{* *}$ \\
\hline $\begin{array}{c}\text { Económica } \\
\mathrm{R}\end{array}$ & & & & & & 1 &, $436^{* *}$ &, $451^{* *}$ &, $345^{* *}$ \\
\hline $\begin{array}{c}\text { Económica } \\
\text { E }\end{array}$ & & & & & & & 1 &, $350^{* *}$ &, $515^{* *}$ \\
\hline $\begin{array}{c}\text { Sexual } \\
\mathrm{R} \\
\end{array}$ & & & & & & & & 1 & ,439*4 \\
\hline
\end{tabular}

Fuente elaboración propia 
Dado que el instrumento utilizado en este estudio permitió observar tanto el reporte de victimización como de perpetración de violencia, un aspecto interesante de analizar es la co -presencia de los distintos tipos de violencia dentro de las parejas, tanto si cada tipo de violencia es recibido como ejercido.

Como se observa en la tabla anterior, en todas las correlaciones los puntajes obtenidos por los participantes en las dimensiones evaluadas son estadísticamente significativas con un $\mathrm{p}<0,01$. Se observa que existen relaciones positivas de magnitud moderada y alta entre las diferentes dimensiones evaluadas.

Un primer análisis es que existe relación directa entre violencia recibida y los otros tipos de violencia. Un segundo análisis posible es que en los casos de violencia psicológica, emocional y física, a mayor frecuencia de violencia recibida, mayor es la frecuencia de violencia ejercida del mismo tipo. La presencia de violencia en las relaciones tiende a manifestarse no bajo una forma de violencia aislada y unidireccional, sino que más bien presentándose simultáneamente en distintas formas.

Las correlaciones más altas se encuentran principalmente entre los tipos de violencia psicológica, emocional y física, tanto recibidas como ejercidas. Por ejemplo, las correlaciones entre violencia física recibida y violencia psicológica y violencia emocional recibidas ( $\mathrm{r}=, 729$ y ,763 respectivamente) están entre las más altas del análisis. Además, también existe una correlación moderada a alta y de dirección positiva entre violencia psicológica recibida y emocional recibida $(\mathrm{r}=, 691)$.

En el análisis de relación entre las violencias ejercidas, cometer violencia psicológica tiene una relación positiva moderada con ejercer violencia emocional y física ( $\mathrm{r}=, 632$ y ,640 respectivamente).

Analizando por separado una bidireccionalidad en cada tipo de violencia, se puede apreciar que recibir y ejercer un mismo tipo de violencia se relaciona en una magnitud alta en los casos de violencia psicológica ( $\mathrm{r}=$ ,753) y violencia emocional $(r=, 740)$ y moderada en el caso de violencia física $(\mathrm{r}=, 653)$.

Ejercer violencia psicológica se relacionó en una magnitud moderada alta con la recepción de violencia emocional y física $(\mathrm{r}=, 613$ y ,609). La perpetración de violencia emocional se relacionó de manera positiva y con magnitud moderada con recibir violencia psicológica y violencia física ( $\mathrm{r}=$ ,503y ,610).

En cuanto a la violencia económica, es interesante que una de las correlaciones más altas del análisis se encuentra entre la violencia emocional ejercida y la económica ejercida $(\mathrm{r}=, 736)$.

En el caso de la violencia de tipo sexual, recibir violencia sexual se 
asoció moderadamente con recibir violencia psicológica $(r=, 525)$ y emocional $(r=, 576)$. Por otro lado, el ejercer violencia sexual se asoció más fuertemente con ejercer violencia emocional $(r=, 645)$. La relación entre violencia sexual recibida y violencia sexual ejercida resultó moderada $(r=, 439)$.

\section{Discusión}

El estudio destaca un alto porcentaje de participantes que reportan haber recibido o ejercido al menos una vez algún tipo de violencia $(85,3 \%$ y $85,6 \%$, respectivamente). Al igual que en otros estudios, los porcentajes más altos de prevalencia se encontraron para la violencia psicológica (\%) y emocional, seguidas por la violencia física, sexual y económica. Los porcentajes son comparables con los estudios de Rey-Anacona (Rey-Anacona, Mateus-Cubides y Bayona 2010; Rey-Anacona, 2013) respecto a la perpetración de violencia. Respecto a la violencia económica, ésta es la menos frecuente, sin embargo es en la única en la cual el hombre manifiesta un mayor porcentaje de prevalencia aun cuando no es estadísticamente significativa.

Según el tipo de violencia los resultados indican que las mujeres reportan un porcentaje estadísticamente significativo de prevalencia en violencia psicológica ejercida mayor que el desarrollado por los hombres.

A nivel de violencia emocional los resultados indican que hay diferencia significativa en la prevalencia de perpetración y victimización, siendo las mujeres quienes reportan un mayor porcentaje de recibir y ejercer este tipo de violencia en relación a los hombres.

En relación a la violencia física los resultados indican que hay diferencia significativa en la prevalencia de perpetración y victimización, siendo las mujeres quienes reportan un mayor porcentaje en la recepción y el ejercicio de este tipo de violencia en relación a los hombres.

A nivel de violencia económica los resultados no señalan diferencias significativas en la recepción y el ejercicio.

A nivel de la violencia sexual los resultados indican diferencias significativas, siendo mayor el porcentaje en mujeres.

La frecuencia levemente mayor de agresiones de parte de las mujeres ha sido descrito frecuentemente en la literatura internacional (Straus, 2004; Glass et al.,2003), el cual puede tener distintas explicaciones. En relación al reconocimiento de la violencia, los hombres presentan un menor reporte, siendo las mujeres más sensibles a admitir estas conductas dentro de la relación. Vizcarra y Póo (2011:94) indica “que en los varones habría un sub reporte de estas conductas, mientras que las mujeres estarían más dispuestas a aceptar su responsabilidad, probablemente porque existe menor sanción social frente a la agresión femenina y ellas no percibirían su conducta 
como dañina”. Harris y Knigth - Bohnhoff (1996) encontraron que la agresión femenina es percibida tanto por hombres como mujeres, como menos grave que la masculina. Las mujeres que agreden físicamente suelen expresarlo con más frecuencia a los demás porque tienden a experimentar sentimientos de culpa (Sharpe y Taylor, 1999).

Se observa que las mujeres presentan una mayor frecuencia de violencia psicológica ejercida hacia sus parejas, concordando con investigaciones que señalan mayor prevalencia del sexo femenino en el reconocimiento de actos agresivos de tipo psicológico o verbal (Rubio-Garay, LópezGonzález, Sául y Sánchez-Elvira-Paniagua, 2012). Sin embargo, respecto de violencia psicológica recibida no hay diferencia entre género, en la misma línea de los estudios que reportan valores similares en la victimización psicológica en jóvenes universitarios (Póo y Vizcarra, 2008).

Es interesante lo que sucede con la violencia emocional, que tiene un comportamiento contrario a la violencia psicológica: las mujeres señalan recibir más violencia emocional, pero no hay diferencias en el reporte del ejercicio en este tipo de violencia. Una interpretación posible de esta diferencia con respecto a la violencia psicológica es que la violencia emocional podría considerarse más severa, al conllevar la intención de generar ansiedad, temor o miedo en la víctima a través de amenazas como "Rompió o destruyó algún bien personal tuyo”, “Te amenazó con el puño u otra parte del cuerpo" o "Amenazó con tener una relación con otra persona”. Este comportamiento estaría más vinculado a un patrón de violencia de género en su acepción más tradicional o terrorismo íntimo, en que el hombre ejerce un rol violento a través del uso o amenaza de uso de fuerza para obtener el dominio de la relación. De todas formas, estas diferencias refuerzan la idea de Rey-Anacona (2009) de tratar separadamente la violencia psicológica de la violencia emocional. Por otro lado, las mujeres señalan recibir más violencia física que los hombres, lo que avalaría las nociones sobre violencia desde una perspectiva de género, en que la victima de las formas de violencia más severas son las mujeres. Los hombres cometen más agresiones físicas severas que las mujeres (González, Muñoz-Rivas, Peña, Gámez y Fernández, 2007). Los hombres que ejercen violencia podrían no querer asumir su papel de agresor, negando o minimizando los actos violentos, y sentir temor al rechazo de los demás si informan que agreden a sus parejas (Molidor y Tolman, 1998). En este sentido, se ha planteado que las respuestas dadas en los autoinformes podrían estar sesgadas o influidas por la deseabilidad social (Rubio Garay et al., 2012).

En cuanto a la violencia sexual, la diferencia por género sólo se encuentra respecto al reporte de victimización. Los estudios en esta perspectiva concuerdan con los resultados de esta investigación, donde las mujeres se encuentran más vulnerables a este tipo de maltrato. El que no haya una diferencia estadísticamente significativa entre hombres y mujeres puede responder a un sesgo de deseabilidad social en el autoreporte de este tipo de conductas, pues es menos probable el reconocerse como perpetrador de violencia sexual por parte de los participantes. 
La no diferencia entre hombres y mujeres respecto a la violencia económica ejercida y recibida obtenidos en este estudio contrasta con los resultados de Rey-Anacona et al. (2010; Rey - Anacona, 2013), donde los hombres reportaron, en promedio, ejercer una mayor frecuencia de violencia económica hacia sus parejas.

La fuerte asociación entre violencia psicológica recibida y violencia física recibida concuerdan con los resultados encontrados por Vizcarra y Póo (2011), a las que se puede agregar la asociación con la violencia emocional recibida. Las autoras relacionan esta asociación a que la violencia en la pareja surgiría como una escalada de conductas que empiezan con las conductas de maltrato psicológico, producto de esto se estaría incorporando la violencia como una estrategia valida de resolución de conflictos a nivel de la pareja.

Las altas correlaciones entre dimensiones de victimización y perpetración apuntarían a la presencia de violencia mutua, donde existe una alta probabilidad de que un miembro de la pareja actúe al mismo tiempo como agresor y como víctima. Ejercer violencia ha sido señalado como el mejor predictor de la victimización tanto física como psicológica y sexual (Harned, 2002; Corral, 2009). La bidireccionalidad de la violencia ha sido señalada como el patrón de violencia más común en la población joven (González y Santana, 2001; Straus, 2004), siendo un patrón más frecuente respecto a la población más adulta (Straus, 2011).

No obstante como señala González, Muñoz - Rivas y Graña (2003) es necesario que exista simetría en la violencia física y psicológica en hombres y mujeres para exista las condiciones de una violencia mutua o cruzada, lo que los resultados del presente análisis no pueden visibilizar. Esto es relevante pues gran parte de los estudios concuerdan en que los motivos, el contexto y las consecuencia de la violencia no son simétricos en términos de género, siendo las mujeres las que se encuentran en mayor riesgo de resultar con lesiones producto de agresiones físicas severas (Corral, 2009).

Uno de las tareas pendientes derivadas de este estudio es la necesidad de la reelaboración del instrumento en aquellas dimensiones que presentan confiabilidad más baja y la verificación de un acortamiento del mismo en las dimensiones que presentan alta consistencia interna.

Finalmente la investigación pretendió ayudar a visibilizar la alta perpetración y victimización de la violencia en jóvenes, siendo más preocupante que la muestra se constituyó con aquellos que cursan educación superior en sus distintos niveles, asumiéndose que la educación es un factor protector o reducidor frente a este problema. La invisibilización sólo ayuda a que este fenómeno se constituya en una pauta estable de comportamiento que tiene el riesgo de presentarse y desarrollarse en futuras relaciones de pareja. Esto conlleva la necesidad de realizar intervenciones tempranas en educación superior o en niveles previos del ciclo educativo, implementando programas educativos y desarrollando la resolución no violenta de conflictos también a nivel de las parejas. 


\section{Nota}

${ }^{1}$ Esta investigación es producto del proyecto de investigación DIULA N025/2011 "Estudio de la violencia en las relaciones de pareja de jóvenes que cursan educación superior en la ciudad de Osorno". 


\section{Bibliografía}

Alberdi, I. y Matas, N. (2002), Violencia doméstica. Informe sobre los malos tratos a mujeres en España. Colección Estudios Sociales, España.

Archer, J. (2000), “Sex differences in aggression between heterosexual partners: A meta-analytic review”. Psychological Bulletin, 126(5), 651-680.

Corral, S. (2009), "Estudio de la violencia en el noviazgo en jóvenes universitarios/as: Cronicidad, severidad y mutualidad de las conductas violentas”. Psicopatología Clínica Legal y Forense, 9(1), 29 - 48.

Dion, K.K. y Dion K.L. (1993), “Individualistic and collectivitic perspectives on gender and the cultural context of love and intimacy”. Journal of Social Issues, 49(3), 53-69.

Follingstad, D.R., Rutledge, L.L, Berg, B.J, Hause, E.S. y Polek, D.S. (1990), "The role of emotional abuse in physically abusive relationships". Journal of family violence. 5(2).107-120.

Furman, W. y Flanagan, A.S. (1997), “The influence of earlier relationships on marriage: And attachment perpective”. En W.K. Halford y H.J. Markman (eds), Clinical handbook of marriage and couples interventions. (p.p. 179202). John Wiley \& Sons Inc, Hoboken, New Jersey, Estados Unidos.

Glass, N., Fredland, N., Campbell, J., Yonas, M., Sharps, P. y Kub, J. (2003), “Adolescent dating violence: Prevalence, risk factors, health outcomes, and implications for clinical practice”. Journal of obstetric, gynecologic, \& neonatal nursing, 32(2), 227-238.

González, R. y Santana, J.D. (2001), “La violencia en parejas jóvenes”. Psicothema, 13(1), 127-131.

González Lozano, M.P., Muñoz-Rivas, M.J. y Graña-Gómez, J.L. (2003), "Violencia en las relaciones de pareja en adolescentes y jóvenes. Una revisión”. Psicopatología clínica, legal y forense, 3(3),23-29.

González Lozano, M. P., Muñoz-Rivas, M. J., Peña Fernández, M. E., Gámez, M. y Fernández, L. (2007), “Análisis de las conductas agresivas en las relaciones de noviazgo en una muestra juvenil de la Comunidad Autónoma de Madrid”. Psicopatología clínica, legal y forense, 7(1), 91-111.

Hamed, M.S. (2002), “A multivariate analysis of risk markets for dating violence victimization”. Journal of interpersonal violence, 17(11), 11791197.

Harris, M. B. y Knight-Bohnhoff, K. (1996), “Gender and aggression I: Perceptions of aggression”. Sex roles, 35(1-2), 1-25. 
Johnson, M. P. (2011), “Gender and types of intimate partner violence: A response to an anti-feminist literature review”. Aggression and Violent Behavior, 16(4), 289-296.

Kanin, E.J. (1957), “Male aggression in dating - courting relations”. American Journal of Sociology, 63 (2), 197-204.

Maturana, H. (1997), “Biología y violencia”. En Maturana H., Coddou F., Montenegro H., Kunstmann G. y Méndez C. Violencia en sus distintos ámbitos de expresión. Dolmen Ediciones, Santiago de Chile, 71 -91.

Molidor, C. y Tolman, R.M. (1998), "Gender and contextual factors in adolescent dating violence”. Violence against women, 4(2), 180-194.

Nunnally, J. C. (1987), Teoría Psicométrica. Trillas, Mexico.

Póo, A. y Vizcarra, M. (2008), "Violencia de pareja en jóvenes universitarios”. Terapia Psicológica, 26(1), 81 -88.

Puget, J. y Berenstein, I. (1989), Psicoanálisis de la pareja matrimonial. Paidós, Buenos Aires.

Rey-Anacona, C. (2008), "Prevalencia, factores de riesgo y problemáticas asociadas con la violencia en el noviazgo: una revisión de la literatura”. Avances en Psicología Latinoamericana, 26(1), 81-88.

Ídem (2009), "Maltrato del tipo físico, psicológico, emocional, sexual y económico en el noviazgo. Un estudio exploratorio”. Acta colombiana de Psicología, 12 (2), 27 - 36.

Rey-Anacona, C.A., Mateus-Cubides, A.M. y Bayona-Arévalo, P.A. (2010), "Malos tratos ejercidos por adolescentes durante el noviazgo. Diferencias por sexo”. Revista Mexicana de Psicología, 27(2), 169-181.

Rey-Anacona C. (2013), "Prevalencia y tipos de maltrato en el noviazgo en adolescentes y adultos jóvenes”. Terapia psicológica, 31(2), 143-154.

Rubio-Garay, F., López-González, M., Sául, L.A. y Sánchez-ElviraPaniagua, A. (2012), “Direccionalidad y expresión de la violencia en las relaciones de noviazgo de los jóvenes”. Acción Psicológica, 9(1), 61-70.

Servicio Nacional de la Mujer. (2009), “Análisis de la violencia en las relaciones de pareja jóvenes. Departamento de Estudios y Capacitación”. Documento de trabajo no publicado.

Servicio Nacional de la Mujer. (2012), “Amor sin violencia: prevención en parejas jóvenes”. Departamento de Estudios y Capacitación. Documento de trabajo no publicado.

Serran, G. y Firestone, P. (2004), “Intimate partner homicide: a review of 
the male proprietariness and the self - defense theories”. Aggression and violent behavior, 9(1), 1-15.

Straus, M. A. (2004), "Prevalence of violence against dating partners by female university students worldwide”. Violence against women, 10(7), 790-811.

Straus, M. A. (2011), “Gender symmetry and mutuality in perpetration of clinical-level partner violence: Empirical evidence and implications for prevention and treatment”. Aggression and Violent Behavior, 16(4), 279288.

Sharpe,D. y Taylor, J.K. (1999), “An examination of variables from a social - developmental model to explain physical and psychological dating violence”. Canadian Journal of Behavioral Science. 31(3), 165-175.

Vizcarra, M. y Poo Figueroa, A. (2011), “Violencia de pareja en Estudiantes universitarios del sur de Chile”. Universitas Psychologica 10(1), 89-98

Recibido: 29.04.2014

Aceptado: 15.08.2014 